Bull. Korean Math. Soc. 51 (2014), No. 5, pp. 1357-1368

http://dx.doi.org/10.4134/BKMS.2014.51.5.1357

\title{
DYNAMICAL BEHAVIOR OF A HARVEST SINGLE SPECIES MODEL ON GROWING HABITAT
}

\author{
ZHI LING AND LAI ZHANG
}

\begin{abstract}
This paper is concerned with a reaction-diffusion single species model with harvesting on $n$-dimensional isotropically growing domain. The model on growing domain is derived and the corresponding comparison principle is proved. The asymptotic behavior of the solution to the problem is obtained by using the method of upper and lower solutions. The results show that the growth of domain takes a positive effect on the asymptotic stability of positive steady state solution while it takes a negative effect on the asymptotic stability of the trivial solution, but the effect of the harvesting rate is opposite. The analytical findings are validated with the numerical simulations.
\end{abstract}

\section{Introduction}

The growth of a single species population that evolves according to a logistic law while assuming that species undergoes a random walk may be modeled by the following equation

$$
u_{t}=d \Delta u+r u\left(1-\frac{u}{K}\right), \quad x \in \Omega, t>0,
$$

where $d$ denotes the diffusion coefficient, $r$ represents the intrinsic growth rate and $K$ is the natural carrying capacity of the environment. We take these parameters to be positive constants. $\Omega$ is a bounded subset of $\mathbb{R}^{n}(n \geq 1)$ with smooth boundary $\partial \Omega$. And the environment $\Omega$ is homogeneous (i.e., the diffusion does not depend on $x) . u(x, t)$ is the density of the species at position $x$ and time $t . \quad u_{t}=\partial u / \partial t, \Delta$ denotes the Laplace operator in $\Omega$. Equation (1.1) is often called Fisher's equation after Fisher [3], who proposed the onedimensional version as a model for the spread of an advantageous gene in a population, and it was also studied by Kolmogoroff, Petrovsky and Piscounoff [6], who studied the equation in depth and obtained some of the basic analytical results.

Received December 3, 2012; Revised May 2, 2013.

2010 Mathematics Subject Classification. 35K57, 92C15.

Key words and phrases. growing domain, population model, asymptotic behavior.

This work was financially supported by PRC grant NSFC (61103018) and NSF of Jiangsu Province (BK2012682, 10KJB110011). 
We assume that the species migrates in a domain surrounded by a hostile environment, so we can consider the initial and boundary conditions as

$$
\begin{array}{ll}
u(x, t)=0, & x \in \partial \Omega, t>0, \\
u(x, 0)=u_{0}(x) \geq 0, & x \in \Omega,
\end{array}
$$

where $u_{0} \in C^{2}(\bar{\Omega})$ and $u_{0}=0$ on $\partial \Omega$. The dynamics of (1.1) and (1.2) has been completely studied, see [4]. Here we briefly describe the results which are related to present paper. Let $\lambda_{1}$ be the principal eigenvalue of the problem

$$
\begin{cases}-\Delta \phi=\lambda \phi, & x \in \Omega, \\ \phi(x)=0, & x \in \partial \Omega,\end{cases}
$$

then we state the following theorem.

Theorem 1.1. For (1.1) and (1.2), the following facts hold:

(1) If $0<r \leq d \lambda_{1}$, then there is only one nonnegative steady state solution $u=0$, which is globally asymptotically stable, that is, for any nonnegative nontrivial $u_{0}, \lim _{t \rightarrow \infty} u(x, t)=0$ uniformly.

(2) If $r>d \lambda_{1}$, then there is only one positive steady state solution $u=u^{*}(x)$, which is globally asymptotically stable, that is, for any nonnegative nontrivial $u_{0}, \lim _{t \rightarrow \infty} u(x, t)=u^{*}(x)$ uniformly.

From the point of view of human needs, the exploitation of biological resources and the harvest of population are commonly practiced in fishery, forestry and wildlife management. Concerning the conservation for the long-term benefits of humanity, there is a wide-range of interest in the use of bioeconomic modeling to gain insight in the scientific management of renewable resources like fisheries and forestry. At the same time, harvesting has a strong impact on the dynamic evolution of a population. Hence it is natural to add the harvesting term to the right-hand side of the first equation in (1.1), and the equation would be

$$
u_{t}=d \Delta u+r u\left(1-\frac{u}{K}\right)-h u,
$$

where $h>0$ is a parameter which represents the level of harvesting, $h v$ is the harvesting yield per unit time.

As we know that the conventional theory of harvested populations basing on equations in which the various environmental is treated as fixed domain. In fact, the ecological environment is not always the same in nature, the habitats of species usually changes due to many reasons, for example, some insects live on a growing leaf, some fishes live in an expanding river due to a warming effect, some animals live in desert which is expanding continuously. A natural question arises that how species react to the changing of their habitats. Take this into account, in present paper, we will consider the problem on growing domain.

Indeed, domain growth has been suggested as an important mechanism in pattern formation and election, we refer to $[1,2,5,8,9,10,16]$ and the references therein for more details. However, since the presence of time-dependent 
transport coefficients in the equations which constructed on growing domain leads to difficulty in stability analysis, most of known work was carried out though numerical computation and simulations.

Recently, Tang etc [15] considered a diffusive logistic equation on one dimensional isotropically growing domain with linear growth function and exponential growth function respectively and get the asymptotic behavior of the solution by constructing upper and lower solutions. In this paper, we try to use this method to study the asymptotic behavior of solution to problem (1.2) and (1.3) on $n$ dimensional growing domain.

The organization of this paper is as follows: In Section 2, a general reactiondiffusion equation with domain growth is developed in $n$-dimensional space $\mathbb{R}^{n}$ and then the harvest single species logistic model on an isotropically growing domain is constructed. In Section 3, we restrict our attention to the isotropically growing domain and analyse the asymptotic behavior of solutions. In section 4 , by performing a series of simulations, we illustrate our analytical result. Finally, we give a brief conclusion in Section 5 .

\section{Model on growing domain}

In this section, we first model a general reaction-diffusion equation on growing domain in $\mathbb{R}^{n}$ and then present the single species harvest model on an isotropically growing domain. The approach is as in [1].

Let $\Omega(t) \subset \mathbb{R}^{n}$ be a simply connected bounded growing domain at time $t \geq 0$ with its growing boundary $\partial \Omega(t)$. For any point $x(t)=\left(x_{1}(t), x_{2}(t), \ldots, x_{n}(t)\right)$ $\in \Omega(t)$, we assume that $u(x(t), t)$ is the density of a species, at position $x(t)$ and time $t \geq 0$. According to the principle of mass conservation, we have

$$
\frac{\mathrm{d}}{\mathrm{d} t} \int_{\Omega(t)} u(x(t), t) \mathrm{d} x=-\int_{\partial \Omega(t)} \boldsymbol{J} \cdot \boldsymbol{n} \mathrm{d} S+\int_{\Omega(t)} f(u) \mathrm{d} x,
$$

where $\boldsymbol{J}$ is the flux across the boundary $\partial \Omega(t), \boldsymbol{n}$ is the outward normal vector on $\partial \Omega(t), f(u)$ is the reaction term within the domain. Using the divergence theorem, the above equation becomes

$$
\frac{\mathrm{d}}{\mathrm{d} t} \int_{\Omega(t)} u(x(t), t) \mathrm{d} x=\int_{\Omega(t)}[-\nabla \cdot \boldsymbol{J}+f(u)] \mathrm{d} x .
$$

On the other hand, the growth of domain generates a flow velocity field $\boldsymbol{a}=\left(\dot{x}_{1}(t), \dot{x}_{2}(t), \ldots, \dot{x}_{n}(t)\right)$. Using the Reynold transport theorem to the lefthand side of (2.1) yields

$$
\frac{\mathrm{d}}{\mathrm{d} t} \int_{\Omega(t)} u(x(t), t) \mathrm{d} x=\int_{\Omega(t)}\left[\frac{\mathrm{d} u}{\mathrm{~d} t}+u(\nabla \cdot \boldsymbol{a})\right] \mathrm{d} x,
$$

where $\frac{\mathrm{d} u}{\mathrm{~d} t}$ is the total derivative of $u$, i.e.,

$$
\frac{\mathrm{d} u}{\mathrm{~d} t}=\frac{\partial u}{\partial t}+\nabla u \cdot \boldsymbol{a} .
$$


Hence we can write (2.1) as follow:

$$
\int_{\Omega(t)}\left[\frac{\partial u}{\partial t}+\nabla u \cdot \boldsymbol{a}+u(\nabla \cdot \boldsymbol{a})\right] \mathrm{d} x=\int_{\Omega(t)}[-\nabla \cdot \boldsymbol{J}+f(u)] \mathrm{d} x .
$$

Since $\Omega(t)$ is arbitrary, then the differential equation

$$
\frac{\partial u}{\partial t}+\nabla u \cdot \boldsymbol{a}+u(\nabla \cdot \boldsymbol{a})=-\nabla \cdot \boldsymbol{J}+f(u) \quad \text { in } \Omega(t)
$$

holds for any $(x, t)$. Assume the species undergoes a random walk, the diffusion flux of $u$ follows Fick's law:

$$
\boldsymbol{J}=-d \nabla u
$$

where $d$ is the diffusive coefficient of $u$. Thus the equation (2.2) becomes

$$
\frac{\partial u}{\partial t}+\nabla u \cdot \boldsymbol{a}+u(\nabla \cdot \boldsymbol{a})=d \nabla^{2} u+f(u) \quad \text { in } \Omega(t)
$$

where $\nabla u \cdot \boldsymbol{a}$ is called advection term while $(\nabla \cdot \boldsymbol{a}) u$ is called dilution term.

In most cases, it is difficult to study the properties of solution to $(2.3)$ because of the advection and dilution terms. Let $y_{1}, y_{2}, \ldots, y_{n}$ be fixed cartesian coordinates in fixed domain $\Omega(0)$ such that $x_{1}(t)=\hat{x}_{1}\left(y_{1}, y_{2}, \ldots, y_{n}, t\right)$, $x_{2}(t)=\hat{x}_{2}\left(y_{1}, y_{2}, \ldots, y_{n}, t\right), \ldots, x_{n}(t)=\hat{x}_{n}\left(y_{1}, y_{2}, \ldots, y_{n}, t\right)$. As $t$ varies, the coordinates $x_{1}, x_{2}, \ldots, x_{n}$ change position with time. These positions are then mapped or transformed to a fixed position given by the $y_{1}, y_{2}, \ldots, y_{n}$ coordinates. Under this transformation, we suppose $u$ is mapped into the new function defined as

$$
u\left(x_{1}(t), x_{2}(t), \ldots, x_{n}(t), t\right)=v\left(y_{1}, y_{2}, \ldots, y_{n}, t\right) .
$$

Thus the equation (2.3) can be translated to another form which is defined on the fixed domain $\Omega(0)$ with respect to $y=\left(y_{1}, y_{2}, \ldots, y_{n}\right)$. However, the new equation is also more complicated [11]. To further simplify the model equations (2.3), we assume that domain growth is uniform and isotropic, that is, the growth of the domain takes place at the same proportion in all directions as time elapses. In mathematical terms, $x(t)=\left(x_{1}(t), x_{2}(t), \ldots, x_{n}(t)\right)$ can be described as follow:

$$
x(t)=\rho(t) y, \quad y \in \Omega(0),
$$

where $\rho(t)$ is called growth function subject to $\rho(0)=1$ and $\dot{\rho}(t) \geq 0$ for all $t>0$.

By (2.4) and (2.5), we have

$$
\begin{gathered}
\boldsymbol{a}=\dot{x}(t)=\dot{\rho}(t) y=\frac{\dot{\rho}}{\rho} x, \\
v_{t}=u_{t}+\nabla u \cdot \boldsymbol{a}, \quad \nabla \cdot \boldsymbol{a}=\frac{n \dot{\rho}}{\rho}, \quad \Delta u=\frac{1}{\rho^{2}(t)} \Delta v,
\end{gathered}
$$


where $n$ is the dimension of space. Then (2.3) becomes the following form

$$
v_{t}=\frac{d}{\rho^{2}(t)} \Delta v-\frac{n \dot{\rho}(t)}{\rho(t)} v+f(v), \quad y \in \Omega(0), \quad t>0 .
$$

Then we obtain the following single species harvest problem on the growing domain $\Omega(t)$ :

$$
\begin{cases}v_{t}=\frac{d}{\rho^{2}(t)} \Delta v-\frac{n \dot{\rho}(t)}{\rho(t)} v+r v\left(1-\frac{v}{K}\right)-h v, & y \in \Omega(0), t>0 \\ v(y, t)=0, & y \in \partial \Omega(0), t>0 \\ v(y, 0)=u_{0}(x(0)), & y \in \Omega(0)\end{cases}
$$

\section{Analysis of the asymptotic behavior}

In this section we will study the asymptotical behavior of the solution of (2.6). Though there are many different kinds of typical growth functions, such as linear growth, exponential growth, logistic (or saturated) growth, in a phenomenological sense, the logistic growth is a biologically reasonable growth function, see [14] for more details. For this reason, we consider the following logistic growth function

$$
\rho(t)=\frac{\exp (k t)}{1+\frac{1}{m}(\exp (k t)-1)},
$$

where $k>0$ and $m>1$. Notice that $\rho(t)$ is continuously differentiable on $[0,+\infty)$ and satisfies

$$
\rho(0)=1, \quad \dot{\rho}(t)>0, \quad \lim _{t \rightarrow \infty} \rho(t)=m>1 .
$$

Next we give the following definition of upper and lower solutions of (2.6):

Definition. A function $\tilde{v} \in C^{2,1}(\Omega(0) \times(0, \infty)) \cap C(\bar{\Omega}(0) \times[0,+\infty))$ is called an upper solution of (2.6) if it satisfies

$$
\begin{cases}\tilde{v}_{t} \geq \frac{d}{\rho^{2}(t)} \Delta \tilde{v}-\frac{n \dot{\rho}(t)}{\rho(t)} \tilde{v}+r \tilde{v}\left(1-\frac{\tilde{v}}{K}\right)-h \tilde{v}, & y \in \Omega(0), t>0 \\ \tilde{v}(y, t) \geq 0, & y \in \partial \Omega(0), t>0 \\ \tilde{v}(y, 0) \geq v_{0}(y), & y \in \Omega(0) .\end{cases}
$$

Similarly, $\hat{v}(y, t) \in C^{2,1}(\Omega(0) \times(0,+\infty)) \cap C(\bar{\Omega}(0) \times[0,+\infty))$ is called a lower solution of (2.6) if it satisfies all the reversed inequalities in (3.1).

To prove our main results, we recall the following two lemmas.

Lemma 3.1 (Comparison Principle). Let $v(y, t)$ be a solution of $(2.6), \tilde{v}(y, t)$ and $\hat{v}(y, t)$ are upper and lower solutions of (2.6), respectively. Then $\hat{v}(y, t) \leq$ $v(y, t) \leq \tilde{v}(y, t)$ in $\bar{\Omega}(0) \times[0,+\infty)$. 
Proof. Define $w=\tilde{v}-v$, and it is easy to see that $w(y, t)$ satisfies

$$
\begin{cases}w_{t} \geq \frac{d}{\rho^{2}(t)} \Delta w-\frac{n \dot{\rho}(t)}{\rho(t)} w+r w\left(1-\frac{\tilde{v}+v}{K}\right)-h w, & y \in \Omega(0), t>0, \\ w(y, t) \geq 0, & y \in \partial \Omega(0), t>0 \\ w(y, 0) \geq 0, & y \in \Omega(0) .\end{cases}
$$

Applying the maximum principle leads to

$$
w(y, t) \geq 0, y \in \Omega(0), t \geq 0,
$$

that is $\tilde{v}(y, t) \geq v(y, t), y \in \Omega(0), t \geq 0$. Similarly, $\hat{v}(y, t) \leq v(y, t)$ can be proved.

Lemma 3.2. Let $v(y, t)$ be a nonnegative nontrivial solution of the following problem

$$
\begin{cases}v_{t}=\frac{d}{\rho^{2}(t)} \Delta v-\frac{n \dot{\rho}(t)}{\rho(t)} v+r v\left(1-\frac{v}{K}\right)-h v, & y \in \Omega(0), t>0 \\ v(y, t)=0, & y \in \partial \Omega(0), t>0 \\ v(y, 0)=v_{0}(y) \geq 0, & y \in \Omega(0) .\end{cases}
$$

If $v(y, 0) \in C^{2}(\bar{\Omega}(0)), v(y, 0)=0, \Delta v(y, 0)=0$ for $y \in \partial \Omega(0)$ and $\Delta v(y, 0) \leq 0$ in $\bar{\Omega}(0)$, then $v(y, t) \in C^{2,1}(\bar{\Omega}(0) \times[0,+\infty))$ and $\Delta v(y, t) \leq 0$ for $y \in \Omega(0)$, $t>$ 0 .

Proof. Since the initial function $v_{0}$ is smooth and satisfies the consistency condition:

$$
\frac{d}{\rho^{2}(0)} \Delta v_{0}-\frac{n \dot{\rho}(0)}{\rho(0)} v_{0}+r v_{0}\left(1-\frac{v_{0}}{K}\right)-h v_{0}=0 \text { for } y \in \partial \Omega(0),
$$

then the standard parabolic regularity theory [7] shows that the solution $v(y, t)$ $\in C^{2,1}(\bar{\Omega}(0) \times[0,+\infty))$. Denote $w=\Delta v$, simple calculations show that it satisfies

$$
w_{t} \leq \frac{d}{\rho^{2}(t)} \Delta w+\left[-\frac{n \dot{\rho}(t)}{\rho(t)}+r\left(1-\frac{2 v}{K}\right)-h\right] w .
$$

Taking into account the condition $\Delta v(y, 0) \leq 0$ we derive $w(y, 0) \leq 0$ for $y \in \Omega(0)$. Since $v(y, t)=0$ for $y \in \partial \Omega(0)$, we have

$$
w(y, t)=\frac{\rho^{2}(t)}{n}\left[v_{t}+\frac{n \dot{\rho}(t)}{\rho(t)} v-r v\left(1-\frac{2 v}{K}\right)+h v\right](y, t)=0, y \in \partial \Omega(0) .
$$

Using the comparison principle gives that $w(y, t) \leq 0$ for $y \in \Omega(0), t>0$, which implies that $\Delta v(y, t) \leq 0$ for $y \in \Omega(0), t>0$.

Let $\lambda_{1}$ be the principal eigenvalue of the problem (1.3) replacing $x \in \Omega$ by $y \in \Omega(0)$ then we have the following two main theorems.

Theorem 3.3. If $0<r \leq \frac{d}{m^{2}} \lambda_{1}+h$, then the solution of problem (2.6) satisfies $v(y, t) \rightarrow 0$ uniformly on $\bar{\Omega}(0)$ as $t \rightarrow \infty$. 
Proof. Obviously, $\hat{v}=0$ is a lower solution of (2.6). The remaining task now is to seek the upper solution of (2.6).

To this end, define $\tilde{v}(y, t)$ to be the unique solution of the problem:

$$
\begin{cases}\tilde{v}_{t}=\frac{d}{\rho^{2}(t)} \Delta \tilde{v}-\frac{n \dot{\rho}(t)}{\rho(t)} \tilde{v}+r \tilde{v}\left(1-\frac{\tilde{v}}{K}\right)-h \tilde{v}, & y \in \Omega(0), t>0 \\ \tilde{v}(y, t)=0, & y \in \partial \Omega(0), t>0 \\ \tilde{v}(y, 0)=M \phi(y), & y \in \Omega(0),\end{cases}
$$

where $\phi$ is the corresponding eigenfunction of $\lambda_{1}, M$ is a positive constant. Noting the behavior of the eigenfunction, $\phi^{\prime}(y)<0$ on $\partial \Omega(0)$ for any $v_{0}(y)$ satisfying $v_{0}(y)=0$ on $\partial \Omega$, there is $M$ such that $M \phi(y) \geq v_{0}(y)$, then $\tilde{v}(y, t)$ is an upper solution of (2.6). It follows from the comparison principle that

$$
0 \leq v(y, t) \leq \tilde{v}(y, t), y \in \Omega(0), t>0 .
$$

Since $\Delta \tilde{v}(y, 0)=M \Delta \phi(y)=-\lambda_{1} M \phi(y) \leq 0$, it follows from Lemma 3.2 that $\Delta \tilde{v}(y, t) \leq 0$ for $y \in \Omega(0), t>0$.

On the other hand, taking into account that $\rho(t)$ tends increasingly to $m$, $1 \leq \rho(t) \leq m$ for $t \geq 0, \tilde{v}(y, t)$ satisfies

$$
\tilde{v}_{t} \leq \frac{d}{m^{2}} \Delta \tilde{v}+r \tilde{v}\left(1-\frac{\tilde{v}}{K}\right)-h \tilde{v} .
$$

Now consider the following problem

$$
\begin{cases}\bar{v}_{t}=\frac{d}{m^{2}} \Delta \bar{v}+r \bar{v}\left(1-\frac{\bar{v}}{K}\right)-h \bar{v}, & y \in \Omega(0), t>0 \\ \bar{v}(y, t)=0, & y \in \partial \Omega(0), t>0 \\ \bar{v}(y, 0)=M \phi(y), & y \in \Omega(0)\end{cases}
$$

We may use the comparison principle again to show that $\bar{v}(y, t) \geq \tilde{v}(y, t)$ for $y \in \Omega(0)$ and $t>0$. So

$$
0 \leq v(y, t) \leq \tilde{v}(y, t) \leq \bar{v}(y, t)
$$

Since that $0<r \leq \frac{d}{m^{2}} \lambda_{1}+h$, we have $\bar{v}(y, t) \rightarrow 0$ uniformly for $y \in \bar{\Omega}(0)$ as $t \rightarrow \infty$ by Theorem 1.1. Thus $v(y, t) \rightarrow 0$ uniformly for $y \in \bar{\Omega}(0)$ as $t \rightarrow \infty$.

Theorem 3.4. If $r>\frac{d}{m^{2}} \lambda_{1}+h$, then the solution of problem (2.6) satisfies $v(y, t) \rightarrow v^{*}(y)$ as $t \rightarrow \infty$, where $v^{*}(y)$ is the unique positive solution of

$$
\begin{cases}-\frac{d}{m^{2}} \Delta v=r v\left(1-\frac{v}{K}\right)-h v, & y \in \Omega(0), \\ v(y)=0, & y \in \partial \Omega(0) .\end{cases}
$$

Proof. Since $\lim _{t \rightarrow \infty} \rho(t)=m$, for any $\varepsilon>0$, there exists a $T_{0}>0$, such that $m-\varepsilon \leq \rho(t) \leq m$ for $t \geq T_{0}$. Similarly, $\lim _{t \rightarrow \infty} \frac{\dot{\rho}(t)}{\rho(t)}=0$ implies that for the same $\varepsilon>0$, there exists another $T_{1}>0$, such that $0 \leq \frac{\dot{\rho}(t)}{\rho(t)} \leq \varepsilon$ for $t \geq T_{1}$. 
Set $T_{*}=\max \left\{T_{0}, T_{1}\right\}$ and let $\tilde{v}(y, t)$ denote the solution of the following problem

$$
\begin{cases}\tilde{v}_{t}=\frac{d}{\rho^{2}(t)} \Delta \tilde{v}-\frac{n \dot{\rho}(t)}{\rho(t)} \tilde{v}+r \tilde{v}\left(1-\frac{\tilde{v}}{K}\right)-h \tilde{v}, & y \in \Omega(0), t>T_{*}, \\ \tilde{v}(y, t)=0, & y \in \partial \Omega(0), t>T_{*}, \\ \tilde{v}\left(y, T_{*}\right)=M \phi(y), & y \in \Omega(0),\end{cases}
$$

where $M$ is a sufficiently large constant, $\phi$ is the eigenfunction defined above. It follows from Lemma 3.1 that $\tilde{v}(y, t)$ is an upper solution of $(2.6)$ in $\bar{\Omega}(0) \times$ $\left[T_{*}, \infty\right)$.

As in the proof of Theorem 3.3, since that $\Delta \tilde{v}\left(y, T_{*}\right) \leq 0$ in $\bar{\Omega}(0)$, then $\Delta \tilde{v}(y, t) \leq 0$ in $\bar{\Omega}(0) \times\left[T_{*},+\infty\right)$, which leads to

$$
\tilde{v}_{t} \leq \frac{d}{m^{2}} \Delta \tilde{v}+r \tilde{v}\left(1-\frac{\tilde{v}}{K}\right)-h \tilde{v}, y \in \Omega(0), t>T_{*} .
$$

Then we consider the following problem

$$
\begin{cases}v_{t}=\frac{d}{m^{2}} \Delta v+r v\left(1-\frac{v}{K}\right)-h v, & y \in \Omega(0), t>T_{*}, \\ v(y, t)=0, & y \in \partial \Omega(0), t>T_{*}, \\ v\left(y, T_{*}\right)=M \phi(y), & y \in \Omega(0) .\end{cases}
$$

Clearly, the problem admits a unique solution $\bar{v}(y, t)$, see [13]. Moreover, since that $r>\frac{d}{m^{2}} \lambda_{1}+h$, the result of Theorem 1.1 shows that $\bar{v}(y, t) \rightarrow v^{*}(y)$ as $t \rightarrow \infty$, where $v^{*}(y)$ is the unique positive solution of (3.2). Using (3.3) and (3.4) and comparison principle yields that

$$
\tilde{v}(y, t) \leq \bar{v}(y, t) \text { for } y \in \Omega(0), t>T_{*} .
$$

This implies that

$$
\limsup _{t \rightarrow \infty} v(y, t) \leq v^{*}(y) \text { for } y \in \Omega(0) .
$$

On the other hand, let $\hat{v}(y, t)$ be the solution of the following problem

$$
\begin{cases}\hat{v}_{t}=\frac{d}{\rho^{2}(t)} \Delta \hat{v}-\frac{n \dot{\rho}(t)}{\rho(t)} \hat{v}+r \hat{v}\left(1-\frac{\hat{v}}{K}\right)-h \hat{v}, & y \in \Omega(0), t>T_{*}, \\ \hat{v}(y, t)=0, & y \in \partial \Omega(0), t>T_{*}, \\ \hat{v}\left(y, T_{*}\right)=\delta \phi(y), & y \in \Omega(0),\end{cases}
$$

where $\delta$ is a sufficiently small constant. It is easy to see that $\hat{v}(y, t)$ is a lower solution of $(2.6)$ in $\bar{\Omega}(0) \times\left[T_{*}, \infty\right)$ if $\delta \phi(y) \leq v\left(y, T_{*}\right)$.

Because $\Delta \hat{v}\left(y, T_{*}\right)=-\delta \lambda_{1} \phi(y) \leq 0$, it follows from Lemma 3.2 that $\Delta \hat{v}(y, t)$ $\leq 0$ for $y \in \bar{\Omega}(0), t \geq T_{*}$ and then

$$
\hat{v}_{t} \geq \frac{d}{(m-\varepsilon)^{2}} \Delta \hat{v}+r \hat{v}\left(1-\frac{\hat{v}}{K}\right)-(h+n \varepsilon) \hat{v}, y \in \Omega(0), t>T_{*},
$$

since that $\rho(t)>m-\varepsilon$ and $\frac{\dot{\rho}(t)}{\rho(t)} \leq \varepsilon$ for $t \geq T_{*}$. 
Next, let us consider the following problem

$$
\begin{cases}\hat{v}_{t}=\frac{d}{(m-\varepsilon)^{2}} \Delta \hat{v}+r \hat{v}\left(1-\frac{\hat{v}}{K}\right)-(h+n \varepsilon) \hat{v}, & y \in \Omega(0), t>T_{*}, \\ \hat{v}(y, t)=0, & y \in \partial \Omega(0), t>T_{*}, \\ \hat{v}\left(y, T_{*}\right)=\delta \phi(y), & y \in \Omega(0) .\end{cases}
$$

Similarly (3.6) admits a unique positive solution, denoted by $\hat{v}_{\varepsilon}(y, t)$. Using comparison principle yields that $\hat{v}_{\varepsilon}(y, t) \leq \hat{v}(y, t)$. Since $r>\frac{d}{m^{2}} \lambda_{1}+h$, we can choose $\varepsilon>0$ sufficiently small such that $r>\frac{d}{(m-\varepsilon)^{2}} \lambda_{1}+h+n \varepsilon$. we then have $\hat{v}_{\varepsilon}(y, t) \rightarrow \hat{v}_{\varepsilon}^{*}(y)$ as $t \rightarrow \infty$, where $\hat{v}_{\varepsilon}^{*}(y)$ is the unique positive solution of

$$
\begin{cases}-\frac{d}{(m-\varepsilon)^{2}} \Delta \hat{v}=(r-n \varepsilon-h) \hat{v}-\frac{r}{K} \hat{v}^{2}, & y \in \Omega(0), \\ \hat{v}(y)=0, & y \in \partial \Omega(0) .\end{cases}
$$

Therefore we have

$$
\liminf _{t \rightarrow \infty} v(y, t) \geq \hat{v}_{\varepsilon}^{*}(y) \text { for } y \in \Omega(0) .
$$

By the continuous dependence of $\hat{v}_{\varepsilon}^{*}(y)$ on $\varepsilon$, we can easily see that $\hat{v}_{\varepsilon}^{*}(y) \rightarrow$ $v^{*}(y)$ as $\varepsilon \rightarrow 0^{+}$. Thus, (3.5), (3.7) and the arbitrariness of $\varepsilon$ imply $v(y, t) \rightarrow$ $v^{*}(y)$ uniformly on $\bar{\Omega}(0)$ as $t \rightarrow \infty$, where $v^{*}(y)$ satisfies problem (3.2). Thus Theorem 3.4 is proved.

\section{Numerical results}

As we know, the presented harvest model is of practical interest only for one or two space variables $(n=1,2)$. In this section, we restrict $\Omega$ to one dimensional space. Numerical simulation is carried out to illustrate the results obtained in previous sections and to explore the long-time behavior of solutions on growing domain. The essential idea of the numerical calculation is to transform the growing domain to a fixed domain as done in equations (2.6). The consequence is that the diffusivity changes from being time-independent on the growing domain to being time-dependent on the fixed domain. On unvaried domain, there are a handful of well-developed numerical methods that can be used directly.

Firstly, regarding the domain growth, we choose $\Omega(t)=[0, x(t))=[0, \rho(t) y)$, where the parameters $k=1, m=2$. It is easy to see that domain grows from initial size $\rho(0)=1$ to the final size $\rho(\infty)=m=2$. We take initial function as $u(y, 0)=\sin (y), y \in(0,1)$ corresponding to $t=0$.

Then, we take the values of the parameters in system (2.6) as follows:

$$
d=0.9, r=2, K=4, h=0.5 \text {. }
$$

In the case of the interval $(0,1), \lambda_{1}=\pi^{2}$. Under the set of parameters in (4.1), we have $0<r<d\left(\frac{\pi}{m}\right)^{2}+h \approx 2.72$. By Theorem 3.3 we know that the solution of problem (2.6) satisfies $v(y, t) \rightarrow 0$ uniformly on $[0,1]$ as $t \rightarrow \infty$. Then we have $u(x, t) \rightarrow 0$ uniformly on any compact subset of $[0,2]$ as $t \rightarrow \infty$. This is 
shown in Figure 1-(a), where the process of domain growth is presented in the left figure. In order to illustrate the result in Theorem 3.4, we choose an other
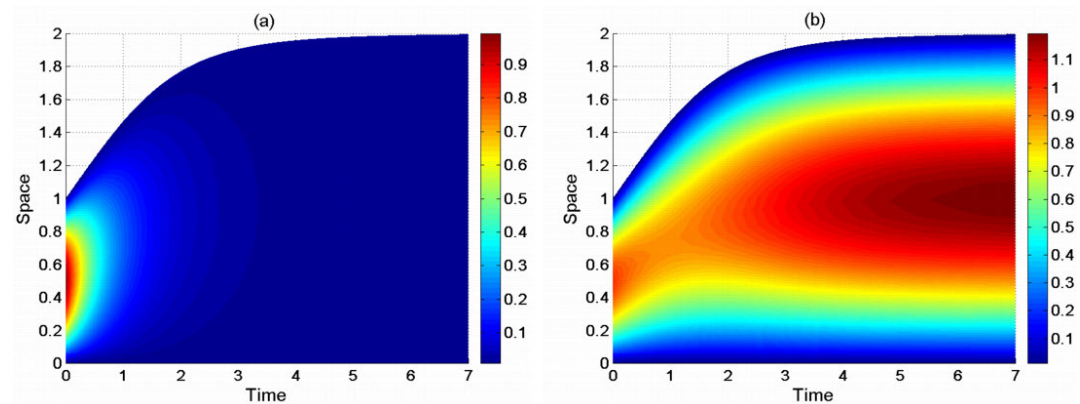

FiguRe 1. Asymptotic behavior of the solution to system (2.6). (a) $r<d\left(\frac{\pi}{m}\right)^{2}+h$, the parameters are given in (4.1). (b) $r>d\left(\frac{\pi}{m}\right)^{2}+h$, the parameters are given in (4.2).

set of the parameters

$$
d=0.9, r=4, K=4, h=0.5 .
$$

In this case, $r>d\left(\frac{\pi}{m}\right)^{2}+h \approx 2.72$, we know that solution $v(y, t)$ of problem (2.6) asymptotically converges to the steady state $v^{*}(y)$ by Theorem 3.4. Figure 1-(b) shows that on the growing domain $(0, x(t))$, the solution $u(x, t)(=$ $u(\rho(t) y, t)=v(y, t))$ asymptotically converges to the steady state $v^{*}(y)$. From the numerical simulation, we can see that most of the individuals aggregate around the center of domain as time increases.

On the other hand, from the Theorem 3.4 we know if $r>d\left(\frac{\pi}{m}\right)^{2}+h$, the species will tend to extinction when the harvesting rate $h$ increases. This can be seen from Figure 2, where we take $h=1$ and 1.5, respectively. The other parameters are same as in (4.2).

\section{Conclusion}

Recently, domain growth is an interesting topic which has attracted a lot of attention. However, most existing results on the long time behaviors of the solutions were investigated through numerical simulations. In this paper, we succeeded achieving the global stability of the solution to a harvest single species logistic model with an isotropic domain growth was studied under Dirichlet boundary condition via upper and lower solutions. We first developed model and then verified the comparison principle which is fundamentally important in studying the asymptotical behavior of temporal solutions to problem (2.6). Then asymptotic behavior of solutions was investigated by approach of upper and lower solutions. Our results show that $v(y, t)(=u(\rho(t) y, t))$ converges to 0 if $r \leq \frac{d}{m^{2}} \lambda_{1}+h$ or to the nonnegative steady state solution if $r>\frac{d}{m^{2}} \lambda_{1}+h$. 

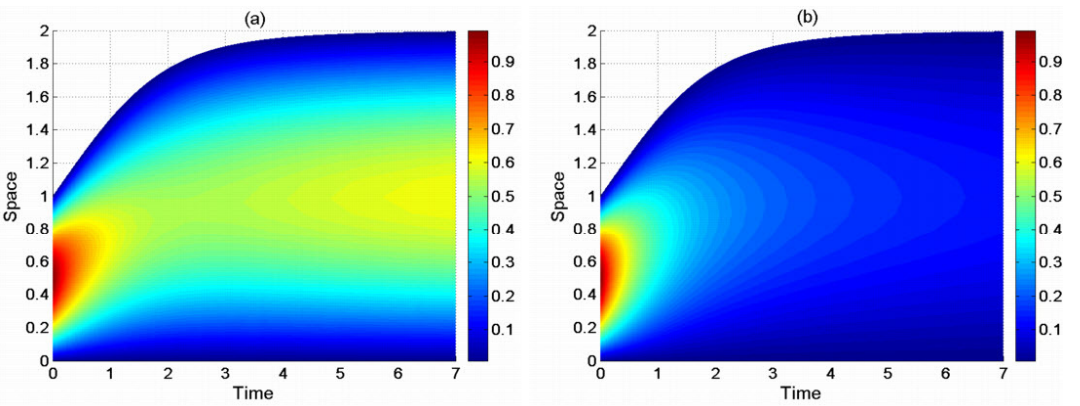

FiguRe 2. Asymptotic behavior of the solution to system (2.6) when $r>d\left(\frac{\pi}{m}\right)^{2}+h$. (a) $h=1$, (b) $h=1.5$, the other parameters are same as in (4.2).

Finally we show that numerical simulations are consistent with our analytical results. Of course, this method allows to obtain the asymptotic estimates for the more general growth functions, which are monotone and continuous differentiable on $[0,+\infty)$.

Ecologically speaking, the results imply that the growth of domain has a positive effect on the asymptotic stability of positive steady state solution and a negative effect on the asymptotic stability of the trivial solution. Conversely, the impact of the harvesting rate is opposite: a negative effect on the asymptotic stability of positive steady state solution and a positive effect on the asymptotic stability of the trivial solution. In other words, if the harvesting rate is large, the species is more at risk for extinction.

\section{References}

[1] E. J. Crampin, E. A. Gaffney, and P. K. Maini, Reaction and diffusion on growing domains: scenarios for robust pattern formation, Bull. Math. Biol. 61 (1999), no. 6, 1093-1120.

[2] E. J. Crampin, W. W. Hackborn, and P. K. Maini, Pattern formation in reactiondiffusion models with nonuniform domain growth, Bull. Math. Biol. 64 (2002), no. 4, $746-769$.

[3] R. A. Fisher, The wave of advance of advantageous genes, Ann. Eugenics 7 (1937), 353-369.

[4] D. Henry, Geometric theory of semilinear parabolic equations, Lecture Notes in Mathematics, 840. Springer-Verlag, Berlin-New York, 1981.

[5] G. Hetzer, A. Madzvamuse, and W. X. Shen, Characterization of turing diffusion-driven instability on evolving domains, Discrete Contin. Dyn. Syst. 32 (2012), no. 11, 39754000.

[6] A. Kolmogoroff, I. Petrovsky, and N. Piscounoff, Study of the diffusion equation with growth of the quantity of matter and its application to a biological problem, (French) Moscow Univ. Bull. Math. 1 (1937), 1-25.

[7] O. A. Ladyzenskaja, V. A. Solonnikov, and N. N. Ural'ceva, Linear and Quasilinear Equations of Parabolic Type, Amer. Math. Soc., Providence, RI, 1967. 
[8] S. Seirin Lee and E. A. Gaffney, Aberrant behaviours of reaction diffusion selforganisation models on growing domains in the presence of gene expression time delays, Bull. Math. Biol. 72 (2010), no. 8, 2161-2179.

[9] J. A. Mackenzie and A. Madzvamuse, Analysis of stability and convergence of finitedifference methods for a reaction-diffusion problem on a one-dimensional growing domain, IMA J. Numer. Anal. 31 (2011), no. 1, 212-232.

[10] A. Madzvamuse, Stability analysis of reaction-diffusion systems with constant coeffcients on growing domains, Int. J. Dyn. Syst. Differ. Equ. 1 (2008), no. 4, 250-262.

[11] A. Madzvamuse, E. A. Gaffney, and P. K. Maini, Stability analysis of non-autonomous reaction-diffusion, J. Math. Biol. 61 (2010), no. 1, 133-164.

[12] J. D. Murray, Mathematical Biology I: An Introduction, Springer, Berlin, 2002.

[13] C. V. Pao, Nonlinear Parabolic and Elliptic Equations, Plenum Press, New York, 1992.

[14] R. G. Plaza, F. Sáchez-Garduño, P. Padilla, R. A. Barrio, and P. K. Maini, The effect of growth and curvature on pattern formation, J. Dynam. Differential Equations 16 (2004), no. 4, 1093-1214.

[15] Q. L. Tang, L. Zhang, and Z. G. Lin, Asymptotic profile of species migrating on a growing habitat, Acta Appl. Math. 116 (2011), no. 2, 227-235.

[16] C. Varea, J. L. Aragón, and R. A. Barrio, Confined turing patterns in growing systems, Phys. Rev. E 56 (1997), no. 1, 1250-1253.

ZHI LING

School of Mathematical Science

YANGZHOU UNIVERSITY

JiangSu Yangzhou 225002, P. R. China

E-mail address: zhling@yzu.edu.cn

LAI ZHANG

Department of Mathematics and Mathematical Statistics

UMEA UNIVERSITY

SE-90187, Ume̊̊ Sweden

E-mail address: zlhometown@126.com 